\title{
New Science Building and Research: From Systems Biology to Theoretical Informatics
}

\author{
Jun You" and Zongrong $\mathrm{Li}^{* *}$ \\ *youjunx@163.com, Editorial Department of the Journal of Hubei University, Wuhan, China \\ **z zrli@hubu.edu.cn, Social Information Science Institute, Huazhong University of Science and Technology, \\ Wuhan, China
}

\begin{abstract}
Briefly introduced the status and related theories foundation of Theoretical Informatics (TI) and Systems Biology (SB). These two disciplines were compared and analyzed in the three aspects of the background, epistemological approach, informational energy of open systems, thus demonstrating the similar attributes or characteristics of the two disciplines, and indirectly discoursing the necessity, the rationality, the feasibility and the vital significance of the establishment and researches for TI. The paper also explores how to draw, reference SB model of development to promote the prosperity and great development of $\mathrm{TI}$.
\end{abstract}

Keywords: theoretical informatics, systems biology, development patterns, system, emergence theory, information, informational energy

Acknowledgement: This belongs to the Ministry of Education, "985 Project", Huazhong University of Science, "Scientific and Technological Progress and Human Spirit" National Social Science Research Base for Innovation, task number: 985hust-0013.

While exchanging greetings with Professor Zongrong Li, the first author discussed Li's Theoretical Informatics $(\mathrm{TI})$ research and only a few brief introductory remarks were needed to arouse the considerable interest of the first author. The first author asked Prof. Li for some references, in particular his doctoral dissertation (Z. Li, 2004) and post-doctoral thesis $(\mathrm{Z}$. Li, 2008). These two papers are full of forward-thinking ideas, clear views, ample evidence, reasonable proofs, and strict logics. Actually Li's papers do not need much complimentary comments made by the first author, since Li has gained the recognition and praise of his international peers as an outstanding contributor to the theoretical foundations of informatics that will be integral to the development of a worldwide Science of Information.

The first author has also been very concerned about information science, and her doctoral dissertation is about bioinformatics. After reading Li's papers on $\mathrm{TI}$, she associated it with the birth and development of the emerging interdisciplinary area of Systems Biology (SB), and noted that the two have much in common. After a brief communication with $\mathrm{Li}$, the first author decided to join the international research team on $\mathrm{TI}$, with $\mathrm{Li}$ and other domestic and foreign scholars, learning together, exploring the theory of information science.

In this article, we will try to compare and analyze $\mathrm{TI}$ and SB, and indirectly elaborate the necessity, the rationality, the feasibility and the vital significance of the establishment of research for $\mathrm{Tl}$; we will discuss the basic theory, epistemological method and the world outlook of TI so as to promote its prosperity and development.

\section{The Present of Theoretical Informatics and Main Arguments}

Knowledge on information systems consists of a large group of subjects, including two major categories: information science and information philosophy. Information science can be divided into two basic levels: applied informatics and TI (or domain informatics and general informatics). The establishment and the consummation of TI, which is the basic theory of information science, show the development and maturity of information science knowledge systems. TI is a theory that is suitable 
to all information phenomenons and each informatics discipline, it commonly solves concrete domain information problems, and it is the basis and core of information science. In contrast, the research achievements of $\mathrm{TI}$, as a public foundation, are few, and progress is slow. Moreover, various applied informatics face the specific discipline respectively, each has an effect in some concrete information field, and provides the comprehensive knowledge about information technology, so the research is still incomplete due to the lack of theoretical explorations and refinement. Along with societal progress, science and humanities and social science's development call for $\mathrm{TI}$, social ideology transformations and newly established worldviews summon TI. Internationally, there are terms synonymous with TI: Unified Theory of Information (UTI), Foundation of Information Science (FIS), etc. Many scholars, from different countries and various professional fields, are actively involved in this research area.

$\mathrm{Li}$ (2004) contributed to the framework of TI. The paper expounds the position and the role of TI in the whole information science system, explains the significance and nature of Tl's problems, discusses TI's fundamentals in the aspects of world outlook and epistemology, introduces Tl's main concepts, principles, and methodology, Tl's basis of informational philosophy, as well as Tl's theoretical and practical applications, etc. As for the methodology of $\mathrm{TI}$, there are two types of syntheses: one is to integrate the results of natural science, the humanities and social science in an interdisciplinary way; the other is to sum up the accomplishments of domain informatics, $\mathrm{Tl}$ and information philosophy in a cross-level way. In comprehensive study, it pays attention to the vertical integration of information evolution in the universe in the past and to the horizontal comprehension of different domain informatics at present.

Information is the meaning of signals and symbols. We found the duality of information existence, information movement and evolution. Cosmic elements have four Yuan: material, information, material energy and informational energy. Information as a logical non-material existence has non-spatial characteristics with no direct observation. What we can quantify is only the information carrier. Therefore, Tl's goal and answering domain, is an unusual scientific problem, and it is incompatible with existing physical background knowledge, scientific methodology and philosophy ontology. By revealing the limitations of physical paradigm and quantitative methods, it indicated that in the evolution of life information, the role of material energy become weaker, while role of informational energy becomes stronger. On this ground, based on genetic information evolution and an explosive growth of human scientific and cultural knowledge, mutatis mutandis, the three basic laws of thermodynamics comprehensively deduced three laws of informatics: information is not conserved, informational energy keeps growing with the time, and information growth has no upper limit. Among them, the second law is the core of TI.

Similar to the construction of a material knowledge system, the process of constructing the information knowledge system consists of three stages: theology, philosophy and science. Since the 1950s, information science disciplines have developed from the empirical thinking of observation and experiment in applied informatics, to the theoretical thinking for searching the common nature of information, and then to the beginning of philosophical thinking seeking new philosophy ontology as the root of information science. Based on the description of the relationship model between material and information, $\mathrm{Li}$ (2008) mainly expounds the information science worldview, namely, the unity of opposites in the worldview of material and information. The paper first discusses the new science standards for the information era, advocating "correspondence theory", "practical theory" and criticizing "consensus theory", to defend for information science "science"; then puts forward a theoretical model of material and information relationship as "double duality" (all things of the world with material and information duality, but also space and time duality (i.e., the static structure and the dynamic process)), and demonstrates the "two worlds theory" as reasonable. Finally the paper elaborates three distinctive features and eight main principles of an information science worldview (or information worldview for short), in a sense, these characteristics and principles just are opposite and complementary to the material scientific world outlook. 


\section{The Rise of Systems Biology and Rapid Development}

Launched in 1990, the Human Genome Project (HGP) is the first great scientific project in the history of life sciences; the plan is an important turning point of development of biology. Led by HGP, a number of Omics appear, which have gradually pushed the era of molecular biology into the era of $\mathrm{SB}$, the biology changes from decomposition to integration, and transforms by descriptive science into quantitative predictive science (Kitano, 2002; Yang, 2004). As one of the sponsors of HGP, American scientist Leroy Hood is one of the founders of SB, and he believes that "SB will be the core driving force of 21st century biology and medicine". Based on this belief, Hood established the first Institute for Systems Biology in the world at the end of 1999. He gave SB such a definition: $\mathrm{SB}$ is to study a biological system, all components (gene, protein, metabolite, etc.) composition and interaction between components under specific conditions (Hood, 2003; Hood, James, Michael, et al, 2004).

In March 2002, "Science" published a SB album, and since then other well-known journals have published research papers on SB. On September 23, 2003, Harvard Medical School established the first SB department in the world, and then the California Institute of Technology, Princeton University and so on in succession, have set up SB research institutions. Since 2000, international conferences on SB, were held annually around different themes of new SB technologies, new ideas, as well as research project developments and progress. Chinese scholars also take great attention to SB. After the establishment of the Shanghai Institute of SB in December 2003, other institutions like Shanghai Jiaotong University "System Biomedical Research Center in Shanghai", etc. were established. The Sino-British Conference on SB was held in June 2005 at Zhejiang University (Chang, 2007). SB has not only been recognized by biologists, but also has aroused the interest of a large number of experts outside the field of biology, at the same time, has attracted the attention of governments with various polices and funds supported.

Nevertheless, so far, the precise definition and understanding of SB is not uniform (Kling, 2006). Academician Chen Zhu summarizes that SB should have a few basic features (Z. Chen, 2005): the hypothesis-driven, quantitative research, and high throughput features because of the Omics technology platforms for applications, covering from nano-science to the ecological system in different scales, and having significant interdisciplinary hallmark. The ultimate goal of SB research is to resolve the complexity of life processes, and to discover and reveal the essential law of life activities by using holistic and systematic research methods.

SB mainly studies the modeling and simulation in entity systems (such as organisms, organs and cells), dynamic analysis of biochemical metabolic pathways, signal transduction pathways of various interactions, gene regulatory networks and disease mechanisms (Flintoft, 2010; Geschwind \& Konopka, 2009; Nottale \& Auffray, 2008; Rho, You, Kim, et al, 2008). The primary mission of SB is to describe the system's state and structure. Next, the researcher should carry on the dynamic analysis to the system evolution. In addition, the description of biological systems state with the system science is hierarchical, the description of different levels may be completely different; the analysis of system evolution mechanisms in system science emphasizes the relationship between the whole and the parts, analyzes how to form the performance and functionality of the whole system through the subsystem function, and should find out the relationship between behavior of the system and that of micro-level.

Research on SB includes two aspects. The first is to obtain experimental data, mainly including various Omics technology platform providing biological data, followed by the use of biological models in computational biology. The perfect integration of "Wet" and "dry" is the real test of SB (Q. $\mathrm{Xu}$, Wang, \& Li, 2008). SB integrates a variety of biological information, experimental data, and establishes mathematical models and verifies the perfect model by experiments, and finally forecasts the behavior of biological systems.

SB is not only a major development for a scientific theory of life, but also has very broad application prospects, such as new drug development (Nicholson, Connelly, Lindon, et al, 2002 ), disease prevention and control (Mani, 2010; Ye, 2009), fine germplasm resources cultivation (L. Li, Xian, Li, et al, 2009; Meyer, Arentshorst, El-Ghezal, et al, 2007; Park, Lee, Kim, et al, 2007; Sindelar \& 
Wendisch, 2007; Srivastava \& Varner, 2007) and so on already have received more and more attention by the scientists from various countries. SB research, will not only promote the development of the life sciences and biotechnology, but also has significant and far-reaching impact on the entire economy, society and mankind.

\section{Comparison Between Theoretical Informatics and Systems Biology}

Through comparative analysis, we discovered that $\mathrm{TI}$ and SB have many common features in aspects of their origin, development, research methods and theory system.

\subsection{Similar Background, Adapting to the Development of the Times and Disciplines}

For the history of the development in the natural sciences, this often happens: when the perceptual experience and some aspects of the knowledge of a natural object or phenomenon accumulated to a certain extent, some people may deeply understand the situation and give a comprehensive summary to make great discoveries or inventions, and establish universal theory or make important scientific hypotheses. A new scientific theory or some new technologies shall become mature and be perfected step by step. When this process is completed, a large comprehensive and leap will appear in theory and technology. In the past two thousand years, this has happened many times in natural history, among which large integrated comprehensives appeared 10 times, such as the establishment of Copernicus's heliocentrism, the discovery of relativity and so on. After each comprehensive and leap, the development of natural sciences gave new vitality, and socio-economic development appeared to be new ball game (Luan, 1986).

TI research caters to the needs of scientific development and social progress. Currently, domain informatics has matured, and technology, products and services of the domain informatics have constituted a powerful information market, information industry and information economy, which drives mankind to transform from an industrial society into an information society. Nevertheless, the information society actually does not have its own specific information science knowledge system as its basis. The existing domain informatics cannot reveal the general rule of all information phenomenons because of its special research object, limited way of thinking, as well as the constraints of the paradigm of physics. Now, we shall accomplish the following tasks: as a whole, from the aspect of organic links within the information phenomenon, to study and master the information, to reveal and grasp the fundamental nature of the information phenomenon and basic laws, to create $\mathrm{TI}$ and establish a complete information science, thus laying the foundation for the establishment of the unity of science which is inclusive of material and information.

In the 20th century, research in biology developed from the macroscopic to the microscopic, from morphological, phenotypic description to research on an organism's various elements and their functions by gradual decomposition and refinement. In 1953, Watson and Crick established the DNA double helix model that signaled the transformation from biology to molecular biology. Genetic engineering technology created in the 1970s had greatly accelerated and extended the development of molecular biology. However, since the organism is a complex system, complex biological phenomena can be demonstrated clearly and comprehensively only through the integration of a variety of molecular mechanisms, approaches and networks. Launched in 1990, the HGP began the comprehensive and systematic exploratory study on biology, so the research shifted from decomposition to integration, the framework has changed from a single biological laboratory for big science projects combined with the model of traditional biological laboratory. In 1994, Wilkins and Williams from Macquarie University, Australia, first proposed the concept of proteome. In 2003, the genome sequencing of human and various model organisms were completed, which for the first time revealed the code of human life. The overlapping of biology with mathematics, physics and computer science resulted in the development of biology from the descriptive to quantitative prediction. HGP and subsequent development of various Omics technologies (mainly genomics, transcriptomics, proteomics, metabolomics, interactionomics, and phenotypeomics, etc.) have gradually pushed the era of molecular biology to the era of SB (Yang, 2004). 


\subsection{Consistent Epistemology Methods, from Reductionism to Emergence}

Bertalanffy divided the overall characteristic of a thing into two: cumulative (additivity) and constitutive (non-additivity) (Guo, 2003). Systems, having the additive property, may usually return to their original state, but the systems with the constitutive character mostly may not return to their original state, that is to say, the use of reductionist methods is restricted. System irreducibleness ubiquitously exists in nature, especially in life, society, and areas of thinking. Once the various parts form the system in a certain way, we can have the attributes, characteristics, behaviors and functions, which the partial or the sum of the partial do not have; once the whole returns to original disparate parts, these properties, characteristics, behaviors, functions, etc., will cease to exist. This thing (or nature), which the whole has and the parts do not have, is called the "Emergent Property", or the "Emergence" (Miao, 2001). The method, which can really provide revision and the supplement for reductionism, is not the whole theory, but emergence theory, or a scientific methodology about emergence.

The main method, which is used in information science to achieve its empirical goal, is emergence theory, that is, the pattern or the model emerged in the discovery of a new nature through observation, experiment, hypothesis and inference. The core concepts and ideas of Emergence are what material elements and information elements constitute a "structural model" of a system. Here, the structure includes hardware and software, which can be spatial structure or temporal structure, can also be a logical structure or algorithmic structure. In the physical world, the structure model of material is a decisive factor in the characteristics of a system. In the world of information, the success and efficiency of communication, control and calculation, without exception, depend on the structural model of the whole formed by system elements, including the hardware, but mainly a software structure model. In a particular information discipline, these structural models often become the cores of all knowledge. Basic concepts, principles and methods of these structural models have become the theoretical foundation of the discipline, and even become common ideas and beliefs, which is the paradigm of the discipline. The basic paradigm shared by information science is called information science paradigm.

Wiener, an American scientist and the founder of Cybemetics, is one of the pioneers who advocated to understand the phenomena of life, from the aspect of system science, and his research led to the birth of biological control theory (Weiner, 1948). In 1969, Bertalanffy proposed General Systems Theory, and he pointed out in his article that organisms is an open system, the comprehensive research on its composition and biological function will eventually need the help of computers, engineering and other branches to complete (Dickson \& Moser, 2007). At that time, since the research tools were far from mature, the theory was not widely accepted by people. As life science research develops into the "Post-genome" era, a broad variety of Omics research represented by Genomics was carried out, new large scientific works appeared, biology began to interlap with the other disciplines such as mathematics and engineering; besides, since modern medicine faced great challenges of a variety of complex multi-gene disease, the idea of using multidisciplinary research method in a systematic way to study biological problems was re-ignited and quickly pushed to the forefront of life science research .

$\mathrm{SB}$ is also known as Integrative Biology or Predictive Biology (Liu, 2005). In the Post-genome era, biology faces a great challenge: how to reveal various biological phenomena through the interaction of DNA, RNA, proteins and small molecules from the overall level of biological systems. At present, it is widely accepted in the scientific community that the phenomenon of life cannot be explained thoroughly by analyzing the composition of the basic units (genes, proteins and biochemical metabolites), and need to be studied through the composition of the system from the interaction of basic units and their dynamic behavior in order to finally answer scientific issues on speciation and the implementation of life functions. Systems biology was born in this context. Compared to traditional biology, which only analyzes the basic unit of biological systems, SB puts more emphasis on the systems level in living organisms to carry out research on life phenomena, that is, taking genes, proteins and metabolites in biochemical reactions for the formation of interconnected nodes, form the network with dynamic characteristics in order to explore and explain complex life 
phenomena (Brazma, Krestyaninova, \& Sarkans, 2006; Bruggeman \& Westerhoff, 2006; Kirschner, 2005; Newman \& Weissman, 2006).

In fact, the integrity and integration of SB research is full of vitality. The rapid development of modern molecular biology, gene individual analysis and so on, make the scientists gradually aware that the complexity of species or life is not directly related to the number of gene. Therefore, objective researchers need to interpret living systems from a holistic, synthetic point of view. Meanwhile, a variety of low flux, high-throughput experimental biological methods make it possible to conduct research on life-support systems from different aspects and levels such as from genes to cells, to tissues, and to different aspects of individuals. Massive use of accumulating data by these means provides the objective conditions for researchers to explain systematically the life phenomena under the integrative viewpoint (L. Chen, Wang, Fei, et al, 2007). To understand a biological system in the systematic approach should become and is becoming the mainstream of biological research methods, aimed at understanding biological systems from the systems level, with emphasis focused on four areas: system structure identification, system behavior analysis, system control and system design.

\subsection{Open System, Emphasizing the Role of Information Energy}

Information duality theory is the basis of TI. Information has both material characteristics and nonmaterial characteristics. Information is dependent on material carriers absolutely; meanwhile, it has a relative independence to special carriers. Radically, information is immaterial with immaterial characteristics. Immaterial characteristics of information are mainly embodied in its non-spatial characteristics. Information duality and its immaterial characteristics are both the basic views in information science and its basic approach. The duality of information existence determines the duality of information movement. Information movement is composed of two interrelated movement modes: material carrier movement and purely information movement, which are driven by different energies and submit to different movement laws. Basically, the driving force of the information movement is not a material power, but the power of information, called "intelligence". Measurement problems of information are different from that in physics, because information has a nonphysical, abstract and logical existence, and does not have the basic properties of physical objects, such as size, odor and etc., so the process of information can not be expressed by mathematical formulas, and only can be described by programs designed in a certain language.

If Wiener takes things into two parts, separating the information from the material, and defining that information is not material; then we can split energy into two, separating information energy from material energy and put forward that the driving force of information movement essentially is not the force in physics, but the power of the informatics. In the level of human society, knowledge does not mean intelligence, a variety of different types of algorithms and programs are the engine of data processing and knowledge inference. If information processing capabilities, namely information "acting" ability, is called "Information energy", then the energy of the universe can be divided into two types: material energy and information energy. Thus, the "Trialism" of the universe (matter, energy, information) can be replaced naturally by "Quadruplicitism" (material, material energy; information, information energy). As all things are a unity of material and information, information energy and material energy are also unified. As the symbol it is the signal of signals, information energy controls material energy. Biological information energy, even human culture energy, in essence, is energy of logic, but in a sense, it is also physical energy. Physical energy is primary information energy, and information energy is advanced physical energy. The unification and evolution of energy becomes logical theory. The evolution of the universe, ostensibly, is the evolution of material structure and function, but in nature is evolution of the energy composition and effectiveness.

Based on the concept that energy can be divided into material energy and information energy, we propose the concept of information entropy and information negative entropy in order to correspond with the concept of material entropy and material negative entropy, to reveal self-organizing systems is just to use the material negative entropy and information negative entropy, to contain 
the increase of material and information entropy, enhance the complexity of the structure and function to achieve evolution from simple to diverse and complex. The Second Law of Thermodynamics predicts a Heat Death of the universe, and the second law of informatics predicts an informationizing and digital universe. Thus, the development direction of system science disciplines correspondingly has been clear: to achieve information turn, that is, to establish information systems science on the basis of information science and control theory.

Material and energy are the central concepts of material science; information and information energy (that is, knowledge and intelligence) are the center of the whole concept of information science. Quaternion of the universe can be understood and accepted by people with common sense. In human society, consuming energy and raw materials is the prerequisite of material production, and then products can be completed. However, materials substances and physical-chemical resources are less required in information production, it consumes neither the raw information nor information energy. On the contrary, intelligence is a growing type of energy. The non-consumption type of knowledge production and the intelligent unceasing proliferation are the inherent mechanism of explosive growth for the information product. In the information world, resource exhaustion and energy shortages can never come true, and it does not lead to the trend of balance, but rather to unlimited extension of a thriving, high-end intelligence system.

Biological systems display complexity from a variety of space levels. The human genome only has 25000 genes, but can create one hundred thousand kinds of protein (many genes have multiple functions), even if only considering the interaction between proteins in pairs; they may also produce five billion kinds of compounds. Tissues and organs are on the next level of biological complexity. Even for the apparent similarity of the organs such as the liver, the function of it in collaboration with the system is completely different from the behavior of the separate cells that compose the organs (cells are sensitive greatly to the surrounding environment and its interaction with neighboring cells). The whole organism is more complex, that is, it is not just the sum of some organs and cells. Higher levels of biological complexity depend on the level of biological communities. Rules and mechanisms formed by self-selection, biological systems have an extraordinary ability to self-organize and aggregate. Through turnover and renewal by components, biological systems also have strong self-maintenance abilities. Perhaps the characteristic that distinguishes biological systems from other systems is that biological systems can change their gene expression and, through signal transduction and protein modification, adapt to changes of the environment (this adaptation reached a peak in higher organisms) (Lei \& Gu, 2007).

Life phenomenon is a complex system, and SB should be a system science that studies life phenomenon. The components of life are complex and are not a simple stack, but have extensive interactions with each other. These interactions of life are not linear, but woven into a network. This wide-existing interaction network leads to another important feature of life phenomenon's complexity, "order and hierarchy". As the constituents of life have stable interactions, they form an orderly structure, with characteristics of self-organization. Another feature of life complexity is that the system is open and life can evolve in this process. Life is not like a simple "self-steady state" system, which can be stabilized through the regulation-control of negative feedback so as to adapt to external changes. Life is an open system far from equilibrium, through the continual formation of new properties or new features to adapt to challenges or change outside.

Information is the basis of SB, high throughput detection technologies are used to produce "massive" information. Through the analysis or excavation of "massive" information, we can further elaborate the regulatory relations between the components of a network, and then we can control and design the system in a regular way. In the era of pro-molecular biology, biologists consider life as a special "Vitality" of the organism, following the rules non-existent in an inorganic realm for life activities. In the era of molecular biology, the researchers think of life as a sophisticated machine, the genes and proteins run according to the laws of physics and chemistry. In the post-genomic era, scientists think of life as the information carrier, all properties can be obtained from the flow of information. Hood believes that biology should be considered an information science, and this view contains three connotations. First, the core of the biological genome is digital. Since the core of 
biology is digitalized, biology can be completely deciphered. Second, the digitalized core of life is showed as two types of information, the first type is the gene of an encoded protein; the second type is the regulatory networks of controlling gene acts. Obviously, the gene composed of a DNA sequence is digitalized. It is worth emphasizing that the information about gene regulatory network in essence is also digitalized, because transcription factor binding sites controlling gene expression are also nucleotide sequences. Third, biological information is hierarchical in order, and flows along different levels. It should be noted that each level of information provides a useful perspective for understanding the operation of living systems. Therefore, the important task of systems biology is to obtain as much information for each level as possible, and then to integrate them.

According to the viewpoint of system theory, the key of constituting a system is not the component of the system, but the interaction or relationship between the components. The interaction or relationship, in essence, is information. From another perspective, life is an open system that is far from equilibrium, in order to maintain its ordering, life systems must be constantly exchanging energy with the external environment in order to offset the process of entropy production. In the 1940s, physicist Schrodinger pointed out in his book named "What is life?" that life lives on "negative entropy flow", while "negative entropy" is actually another representation of information. Therefore, we can say that a life system is a process of information flow. SB is to study and to reveal the operating laws of this information (S. Xu, 2004).

\section{Prospects of Theoretical Informatics}

Through the above analysis and comparison, we can find TI and SB are strikingly similar in many attributes or characteristics, some almost identical. They are both emerging interdisciplinary fields of physics, chemistry, mathematics, computer science, biology, systems science, and so on, and their research results also promote further development of these disciplines. In future development, they can use their own unique advantages, providing ideas and other help for solving major current problems in the field of medical treatment and health care, the environment and resources, energy and life, thus showing us a beautiful picture of a future society.

Although TI and SB were similar in their early periods, the current development situation is completely different. SB has been widely recognized by academics and the community, a number of major international cooperative research centers have been established and SB has obtained a lot of funds from research institutions. However, $\mathrm{Tl}$ is not so lucky, and has not been fully recognized. Although scholars have started to pay attention to it, $\mathrm{Tl}$ is still a vulnerable paradigm. If it wants to obtain recognition from "mainstream" academics, it must seek points that can be accepted and merged by mainstream academics while developing their own disciplines. More importantly, more achievements, which mainstream academics cannot reach, can show the superiority of $\mathrm{TI}$, and the presence of rationality shall be attained so that $\mathrm{TI}$ may be widely recognized by mainstream academics. Only recognized by the international mainstream academics, information science scholars could have a chance to greatly preach IT theory and practice in the international academic arena and promote the holistic development of TI.

The development of TI can learn from and refer to the model of SB, fighting for international cooperation and grand coalition, establishing joint research centers, and striving for funds and project support. More importantly, it is necessary to create an environment in which scientists from different fields can work together to solve the most challenging scientific problems of information. This multi-disciplinary joint program not only allows scientists to fully understand the complexity of information systems, but it also allows scientists from different fields to share their research ideas. To meet the future applications of information science and the development of the field, some new $\mathrm{TI}$ curriculums should be set up as soon as possible for graduate and undergraduate students to stockpile talented people. These measures will bring information science to a whole new realm.

Science is still going forward, the establishment of scientific theory cannot do without the hypothesis, "as long as the Science is thinking, its form of development is the hypothesis" (Engels, 1984), some of the bold creative hypotheses are infants and prototypes of thereafter insights. Of course, the fundamental purpose of scientific research is not the formation of scientific hypothesis, 
but the arrival at the other side of the truth by testing and certificating scientific hypothesis. Based on the principle whether things reflected by scientific hypotheses and phenomena can be directly observed, the scientific method of hypothesis verification can be divided into direct and indirect verification. In the development of the natural sciences, the vast majority of scientific hypothesis were tested and proved by indirect verification, such as Mendeleev's periodic law validation, validation of Einstein's gravitational wave hypothesis (J. Li, 2002).

As for domain informatics, in addition to Shannon's mathematical theory of communication, the remaining subjects may not now and after verified by a direct method to test and prove their hypothesis, it can only be verified by an indirect method. As for TI, the situation is even more special, because $\mathrm{TI}$ integrates many domain informatics. The findings on $\mathrm{Tl}$, such as information duality, information energy as the fourth element of the universe, are based on hypothesis-led, and use the "hypothesis - deductive method" as the basic method of scientific thinking, and the major modes of reasonable verification are all indirect at present or in the future.

$\mathrm{TI}$, as a new science, is in the embryonic stage, whose research scope, research goals, methods and means, application field are to be refined concretely, but because of the importance for $\mathrm{TI}$ on scientific discovery and social progress, it's no doubt that information science will become an important direction in future developments. TI research at home and abroad is still in the initial stage, in fact it has just started, and academic circles are filled with the atmosphere of "pessimism", thinking that it was too difficult, doubting its feasibility. However, we believe, just as the process in which the information age surpassed the industrial age, TI will have a bright future, and it is possible to achieve a higher level than material science theory. On one hand, the formation of scientific hypothesis of TI needs a cognitive process; on the other hand, the verification of these hypotheses, especially through indirect methods, is a historical process of development. TI has a long way to go, and information researchers, making efforts to explore the mysteries of information, should try to create the current situation for new things, to promote prosperity in academic fields. How to follow the trend of the times, and how to carry out in-depth and detailed studies in information fields, are the problems we should seriously consider.

\section{References}

Brazma, A., Krestyaninova, M., \& Sarkans, U. (2006). Standards for Systems Biology. Nature Rev Genet, 7, 593.

Bruggeman, F. J., \& Westerhoff, H. V. (2006). The Nature of Systems Biology. Trends in Microbiology, 15(1), 45- 50.

Chang, C. (2007). Systems Biology Research Progress. Biological Bulletin, 42(6), 18-21.

Chen, L., Wang, Y., Fei, M., et al. (2007). Systems Biology, Its Position in Science and Technology. Science \& Technology Review, 25(10), 5-9

Chen, Z. (2005). Systems Biology - the Core Driver of Medicine and Biology Development in the 21st Century. World Science, 3, 2-6.

Dickson, B. J., \& Moser, E. I. (2007). Neurobiology of Behaviour. Curr Opin Neurobiol, 17(6), 672- 674.

Engels F. (1984). Dialectics of Nature (Yu, G., et. al, Trans.). Beijing: People's Publishing House.

Flintoft, L. (2010). Systems Biology: Small Genome, Complex Regulation. Nat Rev Genet, 11(1), 3.

Geschwind, D. H., \& Konopka, G. (2009). Neuroscience in the Era of Functional Genomics and Systems Biology. Nature, 461, 908-915.

Guo, L. (2003). Reductionism, Self-organization Theory and Calculation Doctrine. Dialectics of nature 12, 83-87.

Hood, L. (2003). Systems Biology: Integrating Technology, Biology, and Computation. Mechanisms of Ageing and Development, 124, 9-16.

Hood, L., James, R. H., Michael, E. P., et al. (2004). Systems Biology and New Technologies Enable Predictive and Preventative Medicine. Science, 306, 640-643.

Kirschner, M. W. (2005). The Meaning of Systems Biology. Cell, 121, 503-504.

Kitano, H. (2002). Systems Biology: A Brief Overview. Science, 295, 1662-1664.

Kling, J. (2006). Careers in Systems Biology: Working the Systems. Science, 311, 1305.

Lei, J., \& Gu, M. (2007). Systems Biology: a New Perspective of Engineering and Medicine - from the UK Academy of Medical Sciences and the Royal Academy of Engineering Report. World Science, 7, 7-10.

Li, J. (2002). Scientific Methodology Overview. Beijing: Science Press.

Li, L., Xian, M., Li, X., et al. (2009). The Application of Systems Biotechnology in Microbial Strain Improvement. Science \& Technology in Chemical Industry, 17(1), 46-50. 
Li, Z. (2004). Concepts, Pringciples and Methods. Huazhong University of Science and Technology, Wuhan.

$\mathrm{Li}, \mathrm{Z}$. (2008). On the Worldview of Information Science. Huazhong University of Science and Technology, Wuhan.

Liu, E. T. (2005). Systems Biology, Integrative Biology, Predictive Biology. Cell, 121, 505-506.

Luan, Y. (1986). Natural Science Research Methods. Hefei: China University of Science and Technology Press.

Mani, K. (2010). Systems Biology and Personalized Medicine in Cancer. Current Pharmacogenomics and Personalized Medicine, 8(1), 64-72.

Meyer, V., Arentshorst, M., El-Ghezal, A., et al. (2007). Highly Efficient Gene Targeting in the Aspergillus niger kusA Mutant. J Biotechnol, 128(4), 770-775.

Miao, D. (2001). System Science is about the Emergence of Science as a Whole. In G. Xu, J. Gu \& C. Hongan (Eds.), Systems Science and Engineering (2nd ed., pp. 167-182). Shanghai: Shanghai Scientific and Technological Education Publishing House.

Newman, J. R., \& Weissman, J. S. (2006). Systems Biology: Many Things from One. Nature, 444, 561-562.

Nicholson, J. K., Connelly, J., Lindon, J. C., et al. (2002 ). Metabolomics: A Platform for Studying Drug Toxicity and Gene Function. Nat Rev Drug Discov, 1(2), 153-161.

Nottale, L., \& Auffray, C. (2008). Scale Relativity Theory and Integrative Systems Biology: 2 Macroscopic Quantum-Type Mechanics. Prog Biophys Mol Biol, 97(1), 115-157.

Park, J. H., Lee, K. H., Kim, T. Y., et al. (2007). Metabolic Engineering of Escherichia Coli for the Production of L-Valine Based on Transcriptome Analysis and in Silico Gene Knockout Simulation. PNAS, 104(19), 7797-7802.

Rho, S., You, S., Kim, Y., et al. (2008). From Proteomics Toward Systems Biology: Integration of Different Types of Proteomics Data into Network Models. BMB Rep, 41(3), 184-193.

Sindelar, G., \& Wendisch, V. F. (2007). Improving Lysine Production by Corynebacterium glutamicum through DNA Microarray-Based Identification of Novel Target Genes. Appl Microbiol Biotechnol, 76(3), 677-689.

Srivastava, R., \& Varner, J. (2007). Emerging Technologies: Systems Biology. Biotechnol Prog, 23(1), 24-27.

Weiner, N. (1948). Cybernetics or Control and Communication in the Animal and the Machine. Cambridge, Massachusetts: MIT Press.

Xu, Q., Wang, C., \& Li, S. (2008). Systems Biology - A New Area of Life Science. China Medical Herald, 5(26), $20-22$.

Xu, S. (2004). Systems Biology[J]. Journal of Biology, 21(3), 8-11.

Yang, S. (2004). The Progress of Systems Biology. Chinese Academy of Science Bulletin, 19(1), 31-34.

Ye, L. (2009). Systems Biomedicine: the Possible Path to Integrate Traditional Chinese and Western Medicine in Theory and Practice. Science, Technology and Dialectics, 26(1), 37-42.

\section{About the Authors}

Jun You

born in 1969, female, associate professor, Ph. D., research direction: editorial research, biomedical engineering, and informatics.

Zongrong Li

born in 1947, male, professor, Ph.D., research direction: software engineering, theoretical informatics, and information worldview. 